\title{
EFFECT OF PREOPERATIVE ASSESSMENT ON MINIMIZING LIVING DONOR LIVER TRANSPLANTATION DONOR COMPLICATIONS
}

By

\author{
Mahmoud Abd Al-Hady Abd Al-Aziz \\ General Surgery department, Faculty of Medicine, Al-Azhar University, Cairo, Egypt \\ E-mail: $\underline{\text { alnaggar82@yahoo.com }}$
}

\begin{abstract}
Background: In adult Living donor liver transplantation (LDLT), donor safety is of paramount importance, and ideally, there should not be any donor deaths.

Objective: To evaluate the potential donor for Living Donor Liver Transplantation as regard general condition, fitness, liver function and segmental anatomy; and assessment of intra- and postoperative complications up to 6 months post-operatively.

Patients and methods: The present study conducted on 200 consecutive cases of living donor liver transplantation over a period of 7 years (2012 to 2018). All patients were admitted at the Department of Transplantation, International Medical Center, Armed Forces Egypt. The study was prospective and designed to evaluate the donors preoperatively, criteria of selection, possible intra-operative and postoperative complications.
\end{abstract}

Results: One hundred and thirty two donors (66\%) had grade I complications, 60 donors (30\%) had grade II complications, $4(2 \%)$ donors had grade IIIa complication, and $123(6 \%)$ donors had grade IIIb complications. Biliary complications were $12 \%$ ( 24 donors) in the form of biliary leak.

Conclusion: Right lobe donor hepatectomy can be performed with an acceptably low incidence of complications. LDLT using the right lobe provides adequate hepatic mass for most adult transplant recipients and gives recipient results similar to conventional cadaveric transplantation.

Key Words: Liver transplantation, Right lobe, Donor complications.

\section{INTRODUCTION}

The liver is an important organ which performs complex functions including metabolism, synthesis and detoxification. Chronic liver disease is a multi-factorial process leading to instability and derangement of essential functions. If not rapidly reversed, complications will lead to hepatic coma. With additional organ failure, recovery becomes irreversible and leads to a high patient mortality. Broadly, the indications for liver transplantation are classified as chronic liver failure; acute fulminant hepatic failure, primary hepatic malignancy and inborn errors of metabolism (Humar et al., 2019).

Human liver transplantation was begun in the United States in the late 1960s by Dr. Thomas Starzl. Interestingly, the majority of his initial patients were children with end-stage liver disease. Unfortunately, in these early series the one-year survival after liver transplantation in both adults and children was only $19 \%$. This survival rate remained unchanged despite advances in 


\section{MAHMOUD ABD AL-HADY ABD AL-AZIZ}

surgical techniques and new immunosuppressive agents until the late 1970s when cyclosporine introduced. Since then, a dramatic and progressive improvement has occurred in both adult and pediatric liver transplant survival. Currently, accepted absolute contraindications to LT by most programs include HIV positivity, spontaneous bacterial peritonitis (SBP) or other active infection, severely advanced cardiopulmonary disease, extra hepatic malignancy that does not meet cure criteria, active alcohol or substance abuse, and inability to comply with immunosuppression protocols because of psychosocial situations (Shi et al., 2020).

\section{PATIENTS AND METHODS}

The present study was conducted on 200 consecutive cases of living donor liver transplantation over a period of 7 years (2012 to 2018). All patients were admitted at the Department of Transplantation, International Medical Center, Armed Forces, Egypt. The study was prospective and designed to evaluate the donors preoperatively, criteria of selection, possible intra-operative and postoperative complications, follow up in the early postoperative period (six months) and how to prevent and manage complications. All donors were subjected to the following three phases: work up, peri operative period and post-operative period. Each phase has been described in details and analyzed.

Patients were selected according to the protocol of living donor liver transplantation of the liver transplantation unit of the International Medical Center, Armed Forces, Egypt.
A living liver donor has a healthy adult with no chronic diseases, and has the ability to give consent. In order to donate legally, the donor has at least 21 years old, not more than 40 years old in Egypt, compatible blood group, BMI maximum 28 , and steatosis not more than $10 \%$.

\section{Absolute Contraindications:}

BMI > 30, Emotional / Psychological Instability, Substance Abuse, Age over 50, Uncontrolled diabetes, Uncontrolled hypertension, Other clinical conditions potentially impairing patient recovery or increasing post-operative complication risk concerning cardiovascular, respiratory, and metabolic systems.

\section{Relative Contraindications (to be evaluated on an individual basis):}

BMI between 28-30, Age 41-50 years old and heavy smokers.

The evaluation protocol was constructed in a step-by-step fashion and took into account the type and the cost of the tests that needed to be performed. The step by-step evaluation process started with the simplest and most cost-effective blood workup, i.e. blood group, virology and serum biochemistries. Once a potential donor was determined to be compatible with the intended recipient, he or she gave Informed Consent for Organ Donation Surgery, as well as other written education materials. In the presence of negative virology, serum biochemistry values within normal limits and a compatible blood group, the potential donor evaluation was preceded to the next step.

If the potential donor has a medical history consistent with some alcohol intake, the liver biopsy was performed 
first, although it was indicated in all donors in Egypt. On the other hand, if there was a question concerning the liver volume, the CT or MRI volumetry was done first. In this way, critical information was obtained first. The next step also included the initial psychological evaluation. All cases were reviewed and discussed in the already established transplant multi-disciplinary selection committee. The members were the physicians of the department of transplantation, the physicians of the department of hepatology, a psychiatrist, an infectious disease specialist, a nutritionist, and the transplant nurse coordinators. The donors advocate team that was formed by the Living Donor Social Worker and the internal medicine donor advocate physician. The potential donor/recipient pair received during their first interview/visit a brochure explaining the rationale and the characteristics of the surgical procedure. After reading the brochure, the potential donor was interviewed by the transplant surgeon and informed about the characteristics of the procedure, the length of the hospital stay, the time to full recovery, the amount of liver that needed to be respected and the general patterns of liver regeneration in addition to data regarding morbidity and mortality for the donor procedure. The donor was also thoroughly informed concerning the prognosis of the recipient so that she/he was able to make the best decision. After this preliminary visit, the donor signed a first consent that allowed the transplant team to proceed with the evaluation. It was only at the end of the evaluation that a second consent form allowing the actual surgery was signed.
All donors underwent pre-anesthesia evaluation.

\section{Graft assessment:}

A. Assessment of Vascular Anatomy: Triphasic computerized tomography (CT) was used to assess arterial and venous anatomy. Multidetector Computed Tomography (MDCT) computer aided 3D reconstruction analysis (MeVis) was done in the cases. Trans-axial $3 \mathrm{~mm}$ CT scans were obtained before and after injection of contrast agents (120 cc of Omniscan 350 at a rate of $4 \mathrm{cc} / \mathrm{second}$ ) and no oral contrast was used. Post-contrast images are obtained in arterial, portal venous and delayed phases. Coronal views were generated and 3-Dimensional reconstruction views were generated on an independent work station. The anatomy of right, middle and left hepatic veins are assessed in venous phase of triphasic CT. Particular attention was given to the MHV and its tributaries. The Portal vein anatomy was assessed mainly in the portal phase of the triphasic CT. The level of the bifurcation and presence of anomalies as trifurcation were noted since they have an implication in the planning of the surgery. Arterial supply was studied in the arterial phase of triphasic CT and in the arterial reconstruction images. This revealed. Conventional anatomy, replaced or accessory right hepatic arteries.

B. Assessment of Biliary anatomy: Anatomy of the bile ducts influenced the difficulty of the anastomosis in the recipient, and potentially influenced the incidence of postoperative biliary 
complications in the donor. The MRCP was done routinely for all donors.

C. Assessment of Liver volumes: The volume of the liver in its totality and the viable graft and rest liver volumes are determined through the analysis of the images of the CT scan. There was a discrepancy between the predicted preoperative liver graft volume and the actual graft weight obtained after the hepatectomy. This discrepancy was between $5 \%$ and 10\%, although it could be in excess or less than predicted. For this reason, it was good practice to leave a margin of safety especially for the donor and accept donors whose rest liver was calculated to be at least at $35 \%$ of the total liver volume.

All donors underwent percutaneous liver biopsy. Donors with non-alcoholic steatohepatitis < $10 \%$ were accepted, while those with non-alcoholic steatohepatitis $>10 \%$ were rejected.

After the graft anatomy and size were available, a preliminary decision for inclusion of MHV, segment 8 or segment 5 veins was done.

\section{Statistical analysis:}

Data were obtained then tabulated in the form of numbers and percent. ages.

\section{RESULTS}

No complications recorded in the preoperative evaluation, including the imaging exams and liver biopsy. Most complications were grade I, II and treated conservatively. These complications included nausea and vomiting, superficial surgical site infection, plural effusion, and diarrhea. 132 donors (66\%) had grade I complications, 60 donors (30\%) had grade II complications, 4 donor (2\%) had grade IIIa complication and 12 donors $(6 \%)$ had grade IIIb complications. No serious grade 4 or 5 complications were observed. There were no donor deaths; in addition, one donor may have one or more than one complication (Table 1).

\section{Table (1): Complications of the donors}

\begin{tabular}{|c|c|c|}
\hline Complications & No. & $\%$ \\
\hline $\begin{array}{l}\text { Grade I: } \\
\text { - } \quad \text { Nausea \& vomiting }\end{array}$ & 32 & 16 \\
\hline - $\quad$ Pleural effusion & 20 & 10 \\
\hline Pulmonary atelectasis & 12 & 6 \\
\hline $\begin{array}{l}\text { - Transient bile leak treated conservatively and stopped within } \\
1 \text { week }\end{array}$ & 12 & 6 \\
\hline - $\quad$ Diarrhea & 8 & 4 \\
\hline $\begin{array}{l}\text { - Postoperative urinary retention (without necessity of } \\
\text { permanent catheterization) }\end{array}$ & 8 & 4 \\
\hline - $\quad$ Thrombophlebitis (i.v. line) & 28 & 14 \\
\hline Psychiatric (chronic pain) & 8 & 4 \\
\hline Depression & 4 & 2 \\
\hline
\end{tabular}


EFFECT OF PREOPERATIVE ASSESSMENT ON MINIMIZING...

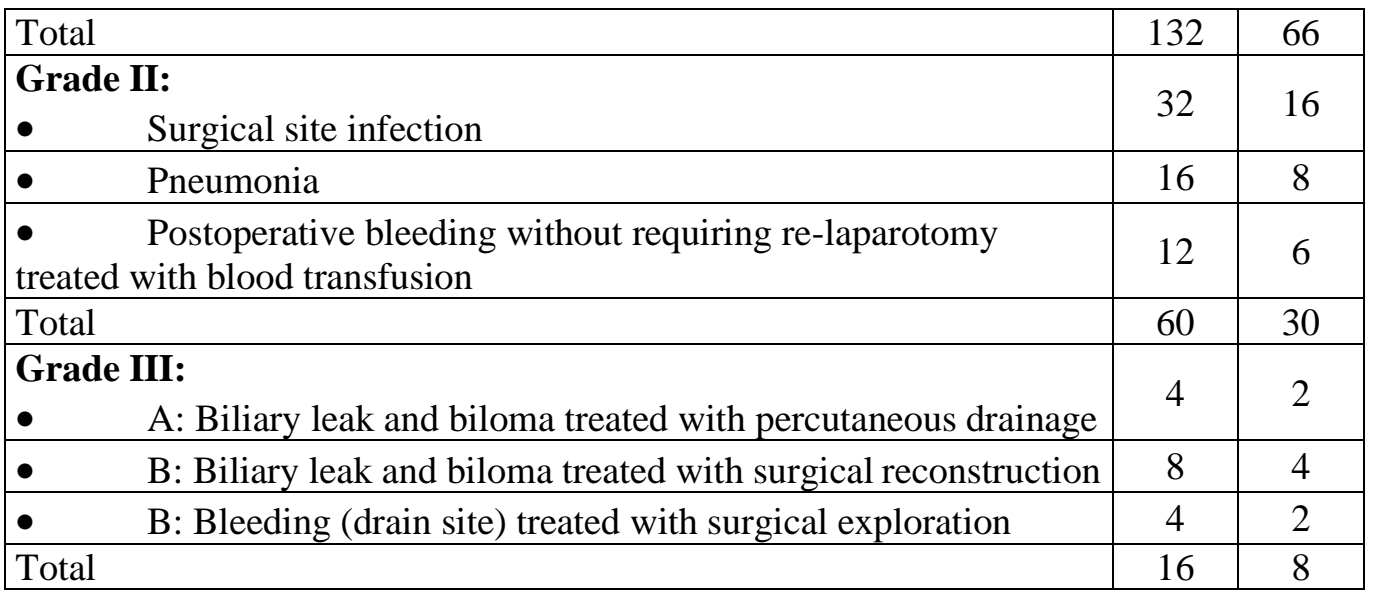

Biliary leak and biloma occurred in 12 donors $(6 \%)$. Four of them had bile leakage exteriorized through pig-tail placed at the site of biloma. The amount of bile drained varied from 70 to $200 \mathrm{cc}$ per day and closed spontaneously within 2 weeks. The others presented later on after removal of the drains by sizable biloma. Trials with ERCP for stenting was done but failed. Surgical correction under general anaethesia was mandatory.

Bleeding from the drain occurred in four cases $(2 \%)$, blood transfusion didn't compensate the loss and the donor needed surgical interference under general anesthesia, the bleeding was from the stump of the right hepatic vein. Resuturing the stump with $4 / 0$ prolene was done (Table 2).

Table (2): Complications of 200 donors classified according the Clavien system

\begin{tabular}{|c|c|c|c|c|c|}
\hline Complications & $\underset{\text { I }}{\text { Grade }}$ & $\begin{array}{c}\text { Grade } \\
\text { II }\end{array}$ & $\begin{array}{l}\text { Grade } \\
\text { IIIa }\end{array}$ & $\begin{array}{l}\text { Grade } \\
\text { IIIb }\end{array}$ & Total \\
\hline Biliary leak & 12 & & 4 & 8 & 24 \\
\hline Bleeding & & 12 & & 4 & 16 \\
\hline $\begin{array}{l}\text { Surgical site } \\
\text { infection }\end{array}$ & & 32 & & & 32 \\
\hline $\begin{array}{c}\text { Pneumonia Pleural } \\
\text { effusion }\end{array}$ & 20 & 16 & & & 36 \\
\hline Total & 32 & 60 & 4 & 12 & 108 \\
\hline
\end{tabular}

\section{DISCUSSION}

Shoreem et al. (2013) defined biliary leakage (BL) regardless the cause of origin, onset, duration, or the volume of drained bile. This extension in the definition could explain the relative high frequency of BL in the present study, any minimal leak for a short period of time that resolved spontaneously was considered as bile leak. Differences in the reported frequency of biliary leakage are probably because of several factors, these factors might be related to the difference in the used technique, team experience or the differences in definition of bile leak.
The reported frequency of bile leak after hepatic resection ranges from $6 \%$ to $14 \%$ (Lafaro et al., 2015).

In Menoufiya National liver institute, biliary complications in the donors were $20 \%$ in the first $140 \mathrm{LDHs}$, and this percentage was reduced to $4 \%$ with adoption of a new technique in the subsequent 50 LDHs (Shoreem et al., 2013).

In Japan, biliary complications occurred in $10 \%$ of patients after right hemiliver resections, compared to only $3.6 \%$ in left hemiliver ones, and $1.9 \%$ in bisegment II-III resections, ten donors 


\section{MAHMOUD ABD AL-HADY ABD AL-AZIZ}

required re-operation for biliary complications (Umeshita et al., 2003). Leaks from peripheral ducts may represent parenchymal leaks overlooked at surgery or may result from sloughing of necrotic tissue from the cut surface (Sakamoto et al., 2016).

In the present study, many leaks are small and typically resolve without intervention and considered as cut surface leaks. Leaks from biliary branches of the caudate lobe are usually refractory, therefore, careful attention should be paid to the performance of continuous suturing and ligation of the bile radicles; this will help to reduce the incidence of bile leakage in both donors and recipients (Shao et al., 2011).

Leaks from major bile ducts, although rare, occurred only in patients in whom the leak presented as an intra-abdominal collection rather than bile in the intraperitoneal drain and may be explained by ischemia of the hepatic duct stump, resulting in necrosis and secondary bile leakage (Sakamoto et al., 2016).

Technical refinements and surgical procedures including the use of a real-time cholangiography by the $\mathrm{C}$-arm, minimized dissection of the hepatic vessels, meticulous closure of the bile duct, and/or the use of Pringle maneuver during the parenchymal transection. Application of these measures reduced the occurrence of BCs from $6.4 \%$ to $1.8 \%$ to $0 \%$ in the last 69 consecutive donors by Taketomi et al., (2010).

Shoreem et al. (2013) advocated a technical improvements to reduce BCs, including closure of the donor biliary stump using interrupted non absorbable sutures and placing of surgical clip below suture line and the use of a real time control cholangiography by the $\mathrm{C}$-arm as a routine, with great reduction of BLs from $18.5 \%$ to $4 \%$ when the new technique was applied.

In a report by Taketomi et al. 2010, the cutting orifice of the hepatic duct has been meticulously closed by 6-0 PDS (Ethicon) continuous suture since 2005 with subsequent reduction of BLs rate. In the present study, the interrupted non absorbable sutures with placing of surgical clip below suture line was applied in 43 donors, only continous non absorbable sutures closure in 6 cases and ligation of long stump in one case. It is important to preserve the blood supply of the biliary tract of both the graft and the liver remnant. Excessive dissection of the hepatic artery and portal vein from the surrounding tissues, results in ischemic change in the biliary tract and occurrence of BCs. (Taketomi et al., 2010).

Meticulous preservation of vascular plexus around bile duct was considered in the present study.

Shoreem et al. (2013) stated that $38 \%$ of BCs after donor hepatectomy managed conservatively without any intervention with obvious success. ERCP offered the advantage of being minimally invasive and provided a method of decompression of the biliary system to allow for closure of leaks, with success rate up to $90 \%$. Half of their cases were managed by ERCP and stent insertion with success rate up to 93\%.

Stent was therefore used to maintain low intrabiliary pressure. Endoscopic stent insertion is safe and effective for treatment of bile leaks after liver resection. A protocol for early endoscopic 
intervention has resulted in rapid closure of the bile leaks and a short hospital stay (Sakamoto et al. 2016). In addition; it is well known that the team experience can lead to improved outcomes. This ongoing developed experience may be a contributing factor in results improvement. Furthermore, Taketomi et al. (2010) explained better outcomes in donors might be related to higher team experiences in addition to the reported technical refinements. The evaluation of suitable donors is related to both donor and recipient safety, the priority is for the donor safety. The volume of the graft should ensure the absolute safety of the donor, but also meet the needs of the recipient. If the graft is too small, it can result in small-for-size graft syndrome. In general, the younger the donor, the better is the liver's regenerative capacity, thus donors aged between 18 and 60 years are required (Masuda et. al., 2004).

In the present study, donors' age ranged from 20 to 40 years for more safety. During the preoperative examination, there were no obvious abnormal liver functions, obvious apparent blood vessels, biliary anatomical abnormalities, or preoperative liver biopsies with serious fatty degeneration. According to the remnant liver volume, the study group was divided into two groups. Data for each group of donors was recorded and included preoperative parameters. No statistical differences between the groups were found. The two groups also had homogeneity of the body, excluding other factors of donor recovery.

In the present study (200 donors), one hundred and thirty two donors $(66 \%)$ had grade I complications, Sixty donors had grade II complications, Four (2\%) donors had grade IIIa complication and Twelve $(6 \%)$ donors had grade IIIb complications. Biliary complications were $12 \%$ in the form of biliary leak, 12 of them $6 \%$ were grade I treated conservatively, 4 case $2 \%$ treated with US guided aspiration with pigtail drainage, and 8 cases $4 \%$ needed surgical reconstruction. Postoperative bleeding occurred in 16 cases $8 \%, 12$ of them $6 \%$ treated conservatively and 4 of them $2 \%$ treated with surgical exploration. Reoperation rate was 6\%. Wound infection rate was $16 \%$. No serious grade 4 or 5 complications were observed. Grade IIIb complications of bile leakage were two right hepatic ducts, and grade IIIa complication was single right hepatic duct. This reflected that increased incidence of leakage associated with multiplicity of stumps of the right hepatic ducts. In the present study, biliary stricture was not recorded up to six months of postoperative follow-up, despite the change in method of closure of biliary stumps from purse string to interrupted sutures with clip.

In the present series medical visualization (MeVis) study was done to most cases (184 cases, 92\%). It gave good idea about segmental anatomy of the liver and relation between vascular and biliary structures thus minimizing the incidence of vascular or ductal injury.

According to a worldwide report in 2016, there had been 19 reported donor deaths mostly due to sepsis and liver failure (Raza et al., 2020). Data from 84 centers showed a donor mortality rate of 0.2\% (Humar et al., 2019). In the present study there were no donor deaths. 


\section{MAHMOUD ABD AL-HADY ABD AL-AZIZ}

\section{CONCLUSION}

Right lobe donor hepatectomy can be performed with an acceptably low incidence of complications. LDLT using the right lobe provides adequate hepatic mass for most adult transplant recipients and gives recipient results similar to conventional cadaveric transplantation.

\section{REFERENCES}

1. Allam N, Al Saghier M, El Sheikh Y, Al Sofayan M, Khalaf H, Al Sebayel M, Helmy A, Kamel Y, Aljedai A, Abdel-Dayem H, Kenetman NM, Al Saghier A, Al Hamoudi W and Abdo AA. (2010): Clinical outcomes for Saudi and Egyptian patients receiving deceased donor liver transplantation in China. Am J Transplant.,10(8):1834-41.

2. Ding Yuan, Yong-Gang Wei, Bo Lis, LuNan Yan, Tian-Fu Wen and Ji-Chun Zhao (2011): Evaluation outcomes of donors in living donor liver transplantation: a single center analysis of 132 donors. Hepatobiliary \& Pancreatic Diseases International. 10:480- 84.

3. Humar, A., Ganesh, S., Jorgensen, D., Tevar, A., Ganoza, A., Molinari, M. and Hughes, C. (2019): Adult living donor versus deceased donor liver transplant (LDLT versus DDLT) at a single center: time to change our paradigm for liver transplant. Annals of surgery, 270(3), 444-451.

4. Lafaro, K., Buettner, S., Maqsood, H., Wagner, D., Bagante, F., Spolverato, G. and Pawlik, T. M. (2015): Defining post hepatectomy liver insufficiency: where do we stand? Journal of Gastrointestinal Surgery , 19(11), 2079-2092

5. Raza, M. H., Kim, M. H., Ding, L., Fong, T. L., Romero, C., Genyk, Y. and Emamaullee, J. (2020): Long-Term Financial, Psychosocial, and Overall Health-Related Quality of Life after Living Liver Donation. Journal of Surgical Research, 253: 41-52.
6. Sakamoto, K., Tamesa, T., Yukio, T., Tokuhisa, Y., Maeda, Y., and Oka, M. (2016): Risk factors and managements of bile leakage after hepatectomy. World Journal of Surgery, 40(1), 18-25.

7. Shao $F$, Wang, Z, Yong Hu and Xiao $P$ C (2011): Biliary complications after living donor liver transplantation. Liver Transplantation, 1127-36.

8. Shoreem H., Hegazy O., Soliman H., Saleh Sh., Yassen T., Aziz A. M., Salama I., Abuelella Kh., Ibrahim T., Marawan I. and Tanaka K. (2013): A new modified technique in closure of bile duct stump in living donor hepatectomies. Egyptian J. Surgery,32(7):118132.

9. Taketomi A., Morita K., Toshima T., Takeishi K., Kayashima H. and Ninomiya M. (2010): Living Donor Hepatectomies with Procedures to Prevent Biliary Complications. J. Am. Coll. Surg., 211: 456-64.

10. Masuda, Y., Yoshizawa, K., Ohno, Y., Mita, A., Shimizu, A., and Soejima, Y. (2020): Small-for-size syndrome in liver transplantation: Definition, pathophysiology and management: SFSS in LT. Hepatobiliary \& Pancreatic Diseases International. 45(9)110115.

11. Lafaro, K., Buettner, S., Maqsood, H., Wagner, D., Bagante, F., Spolverato, G. and Pawlik, T. M. (2015): Defining post hepatectomy liver insufficiency: where do we stand? Journal of gastrointestinal Surgery, 19(11): 2079-2092.

12. Shi, Y., Liu, C., Zhang, H., Huang, Y., Sun, M., Wang, W., and Shang, S. (2020): Changes in the quality of life of living liver donors: A meta-analysis. International Journal of Nursing Studies, 103586. 
دور تقييم ما قبل العمليات الجر احية في تقليل المشاكل الناتجة

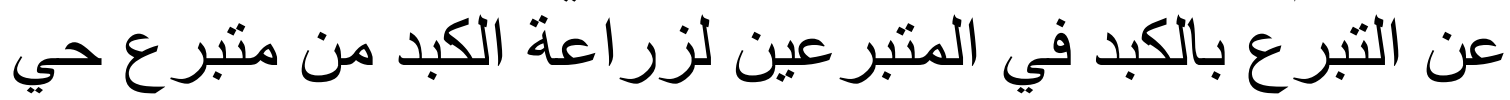
محمود عبد الهادي عبد العزيز

قسم الجراحة العامة، طب الأزهر بالقاهرة

E-mail: alnaggar82@yahoo.com

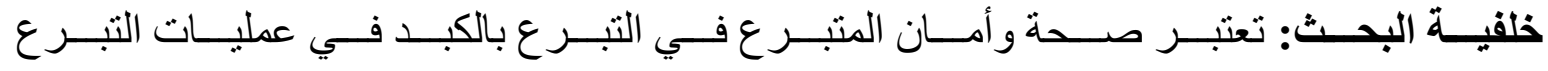

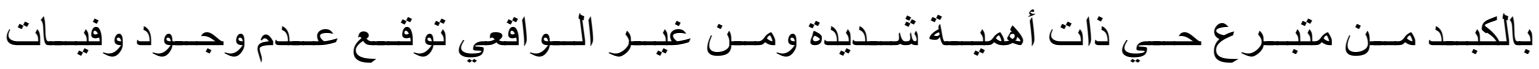

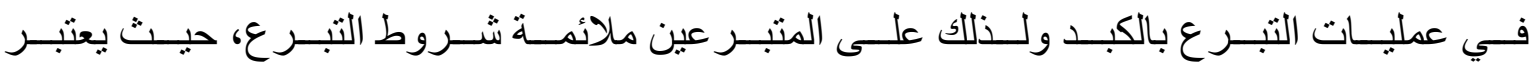

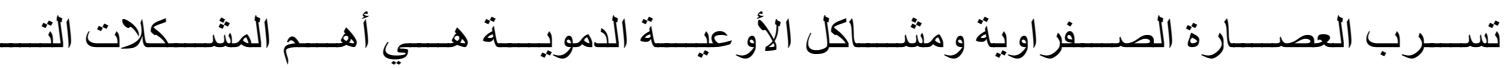
تحدث للمتبرع.

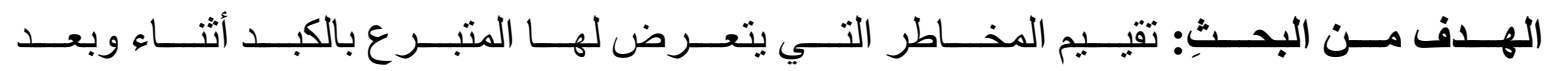
عملية التبر ع بالكبد من متبرع عي.

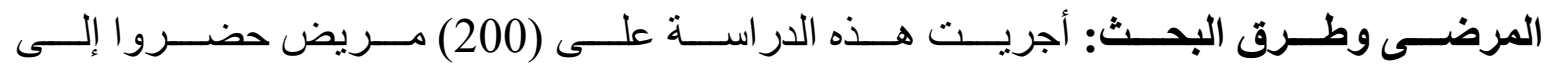

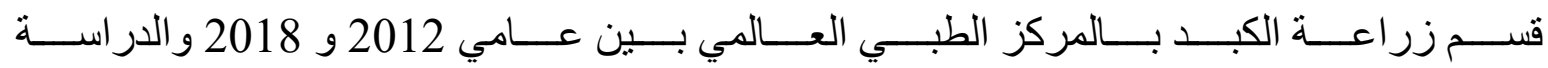
لتقييم إمكانية حدوث مشاكل أثناء وبعد عملية زر اعة الكبد للمتبرع.

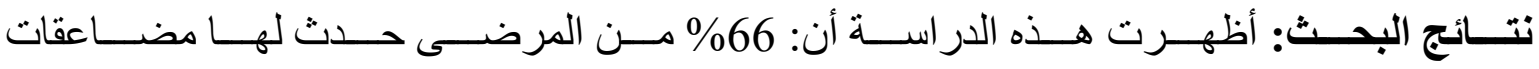

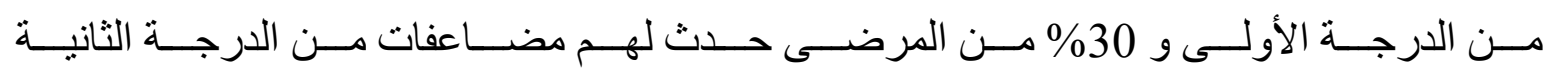

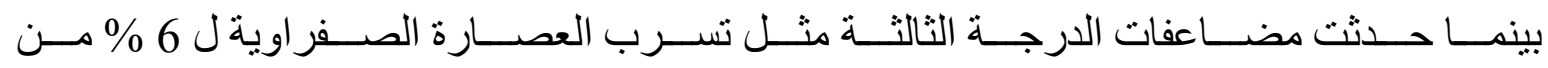
المرضى.

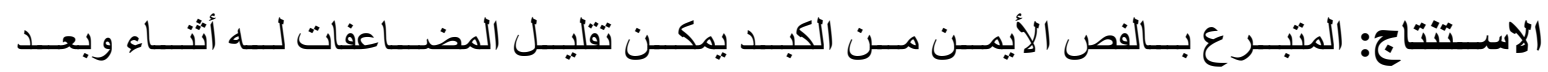

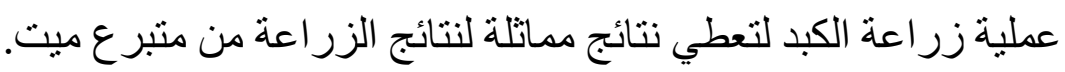

